\title{
Indoor positioning utilizing bluetooth low energy RSSI on LoRa system
}

\author{
Kavetha Suseenthiran, Abd Shukur Ja'afar, Ku Wei Heng, Mohamad Zoinol Abidin Abd Aziz, \\ Azmi Awang Md Isa, Siti Huzaimah Husin, Nik Mohd Zarifie Hashim \\ Center for Telecommunication Research \& Innovation (CeTRI), Faculty of Electronic and Computer Engineering \\ (FKEKK), Universiti Teknikal Malaysia Melaka (UTeM), Hang Tuah Jaya, Melaka, Malaysia
}

\begin{tabular}{l} 
Article Info \\
\hline Article history: \\
Received May 31, 2021 \\
Revised Jul 12, 2021 \\
Accepted Jul 14, 2021 \\
\hline
\end{tabular}

\section{Keywords:}

BLE positioning

Trilateration

Wireless positioning

\begin{abstract}
Indoor positioning systems has become popular in this era where it is a network of devices used to locate people or object especially in indoor environment instead of satellite-based positioning. The satellite-based positioning global positioning system (GPS) signal is affected, and loss incurred by the wall of the building causes the GPS lack of precision which leads to large positioning error. As a solution to the indoor area coverage problem, an indoor positioning based on bluetooth low energy (BLE) and long range (LoRa) system utilising the receive signal strength indicator (RSSI) is proposed, designed, and tested. In this project, the prototype of indoor positioning system is built using node MCU ESP 32, LoRa nodes, and BLE beacons. The node MCU ESP 32 will collect RSSI data from each BLE beacons that deployed at decided position around the area. Then, linear regression algorithm will be used in distance estimation. Next, particle filter is implemented to overcome the multipath fading effect and the trilateration technique is applied to estimate the user's location. The estimated location is compared to the actual position to analyze the root mean square error (RMSE) and cumulative distribution function (CDF). Based on the experiment result, implementing the particle filter reduces the error of location accuracy. The particle filter achieves accuracy with $90 \%$ of the time the location error is lower than 2.6 meters.
\end{abstract}

This is an open access article under the CC BY-SA license.

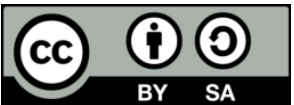

\section{Corresponding Author:}

Abd Shukur Ja'afar

Center for Telecommunication Research \& Innovation

Faculty of Electronic and Computer Engineering, Universiti Teknikal Malaysia Melaka

Hang Tuah Jaya, 76100 Durian Tunggal, Melaka, Malaysia

Email: shukur@utem.edu.my

\section{INTRODUCTION}

Positioning system has been a part of primary element in human daily activities today. The development of positioning system is one of the essential for the development of civilizations of human as it can be used in many sectors like security, military, logistics, monitoring, tracking, health care, sport, and entertainment. GPS is a satellite navigation system used to determine the ground position of an object, especially for outdoor environment while indoor positioning system (IPS) is most common used in inside building or indoor environment. IPS is demand higher accuracy compare than GPS.

Indoor positioning system (IPS) can classify into three major groups which are network-based systems, inertial systems, and hybrid systems [1]. IPS is the system that replace GPS technology to locate the position location of a user or a device in indoor environment [2]. Network based system is known as a radio frequency-based system which build with the wireless technology, utilize the wireless signal to determine the 
position such as wireless-fidelity (WI-FI) based positioning system, BLE beacons based indoor positioning system, RFID indoor positioning system, and other wireless technology system. Inertial based system is use self-contained sensor or inertial sensors to estimate the location of user [3]. The hybrid system is the system which combine two or more method or technique to measure the user position [4]. The system uses several different wireless communication technologies in one system to estimate user's position, such as hybrid indoor positioning that employs both Wi-Fi and bluetooth [5].

Bluetooth low energy technology is a new standard for wireless personal area network technology developed by bluetooth special interest group (SIG) [6]. Due to the low cost, low power consumption, easyto-deploy solution and the vast majority of portable devices in the, BLE beacon has been used for indoor localization [7]. BLE beacon is low power consumption device and its coverage area will become smaller compared to WI-FI due to short signal reachable distance [8]. The beacons can be placed at various places due to its small dimension [9]. BLE beacon operate on $2.4 \mathrm{Ghz}$ frequency which is same with classic Bluetooth and both technologies apply similar modulation scheme which is gaussian frequency shift keying.

RSSI trilateration is the method which highly rely on the RSSI and uses the path loss propagation model to compute the distance between the target position and the transmitter also known as the reference point [10], [11]. Trilateration technique is applied to utilize the point of intersection formed by three circles of BLE beacon's coverage area and the distance to decide the exact position [12], [13]. Distance that is calculated by using path loss model plays an important role in estimating the position of the user [14], [15]. The IPS using trilateration technique is easy to build and low-cost system as the RSSI value are easy to extract by the receiver [16], [17]. The accuracy of the IPS using the trilateration method can be increase if it is combined with other algorithm or technique.

Fazli Subhan and his team conducted their indoor positioning system project in bluetooth network using fingerprinting and trilateration [18], [19]. Bluetooth USB dongles was used as fixed reference node while Nokia mobile devices was used as receiver to collect RSSI data [20]. This experiment collects the RSSI in the lab that area having $120 \mathrm{~m}^{2}$ and the average error of positioning was $2.67 \mathrm{~m}$. Besides that, Vicente Cantón Paterna and his friend conducted a study with using channel diversity where Kalman filter and weighted trilateration is used to form an indoor positioning system [21]. The estimation error for the moving object in two scenario environment room which has an area of $54 \mathrm{~m}^{2}$ and $290 \mathrm{~m}^{2}$ respectively are lower than $1.82 \mathrm{~m}$ and $4.6 \mathrm{~m}$ during $90 \%$ of the time with using only 4 beacons.

\section{METHODOLOGY}

The indoor positioning system based on BLE beacon RSSI data extraction technique is designed and developed to determine the user's position. Based on the system block diagram in Figure 1, this project consists of four process subsystems to perform each function at different stages. First process is setup BLE network with deploy of BLE beacons at each different particular position at broadband \& networking (BBNET) lab and set-up a square shape grids in the area environment where this square shape grid will have multiple access points that is used for collecting RSSI data. At second stage, the node MCU ESP32 will collect the RSSI that is transmitted by each BLE beacons at each multiple access points and user current position. The interchange data process happens between two LoRa nodes to upload and store RSSI data into laptop. Next, is the algorithm development phase where the particle filter is implemented to stabilize data and trilateration technique is used to estimate the position of the user. The output result of this positioning system shows the estimated user's position in 2-d diagram. Subsequently, the accuracy error is calculated between actual and estimated position.

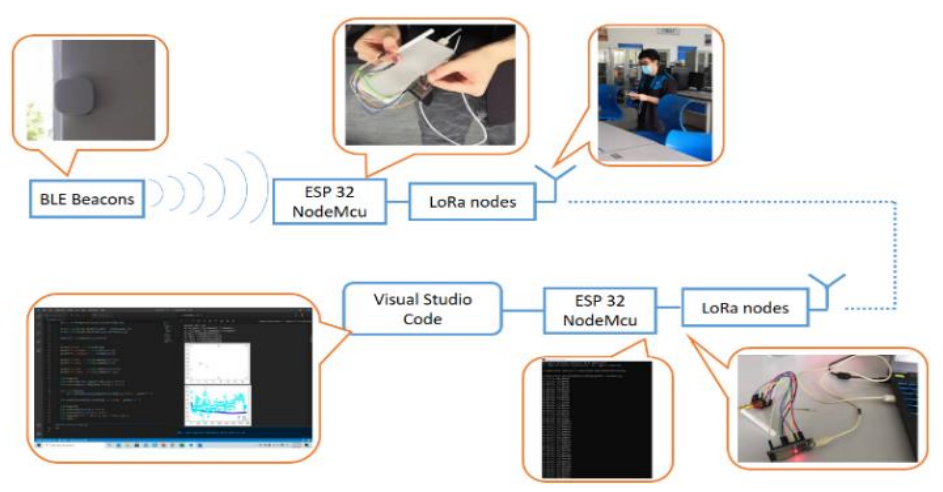

Figure 1. Overview of positioning system set-up 


\subsection{Data collection and set-up procedure}

All the experiment is conducted in BBNET lab, FKEKK. BLE beacon devices is attached on the walls at the height level which is about $1.6 \mathrm{~m}$. Each beacon devices have their own address, so the collected RSSI data are easily classify based on the address. First, a square shape grids in the area environment is setup before the system starts to estimate user's specific position. The whole area that is used for position estimation is divided in square shape grids and their locations are in terms of 2-D plane, XY coordinate. Figure 2 as shows in the set-up of the BLE beacons in the BBNET lab with grid of $2 \mathrm{~m}$.

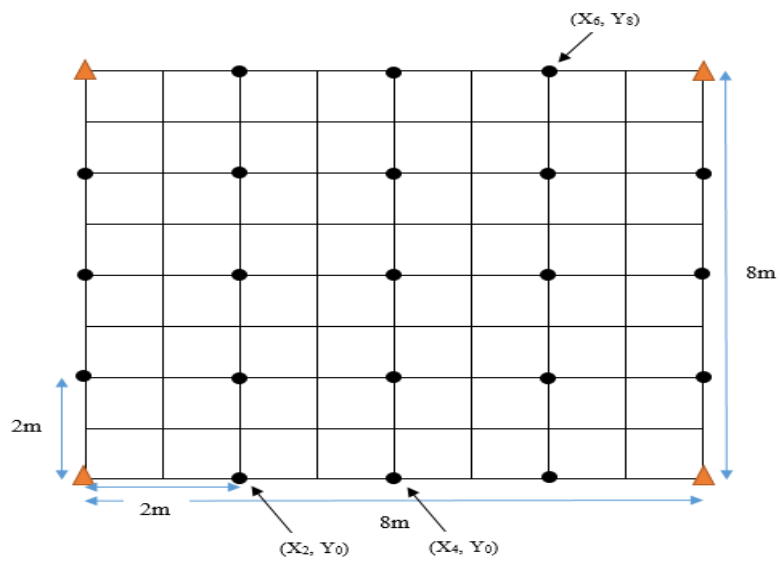

Figure 2. Square shape grids in $8 \mathrm{~m} \times 8 \mathrm{~m}$ area

The black dot points (-) that is shown in Figure 2 represents multiple references points and the position of each black dot points are assumed as $\left(\mathrm{X}_{0}, \mathrm{Y}_{0}\right),\left(\mathrm{X}_{2}, \mathrm{Y}_{0}\right) \ldots\left(\mathrm{X}_{\mathrm{n}}, \mathrm{Y}_{\mathrm{n}}\right)$. The triangle shape points $(\triangle)$ are the position for each BLE beacons with known coordinate point value. Each multiple reference point will conduct RSSI collection within 2 minutes by using node MCU ESP32. The purpose for RSSI collection in the square shape grids is to find the parameters which are $m$ (Gradient) and $c$ (intercept) in the formula (4) by using linear regression method. There are two parts for Node MCU ESP32 connect with LoRa nodes:

- Transceiver part: node MCU ESP32 is connected to LoRa module that which will be the mobile part. User will be place at particular position around the area. Then, the Node MCU ESP32 will receive and collect bluetooth's RSSI that is transmitted by each BLE beacons. Next, LoRa nodes will send this bluetooth's RSSI that is collected by node MCU ESP32 to another LoRa node at the receiver part.

- Receiver part: consists of one set of node MCU ESP32 connected with LoRa node. The LoRa node in here will receive the RSSI data that sent by LoRa node from the user. Then the node MCU ESP32 at receiver is connected to the laptop for communicate with Python based code to collect importance parameter such as bluetooth's RSSI and UUID.

Figure 3(a) and 3(b) is being as respectively shows a transceiver ESP32-LoRA (with 3 BLE beacons in experiment) and a receiver LoRA node connected to a laptop.

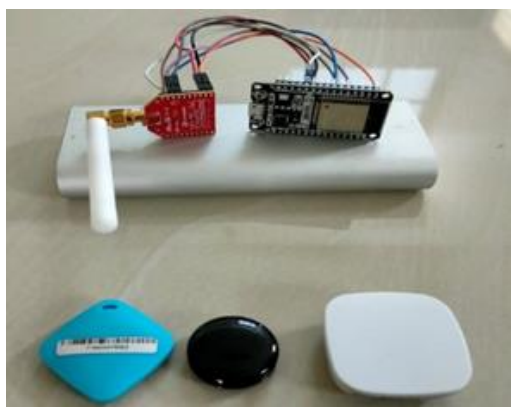

(a)

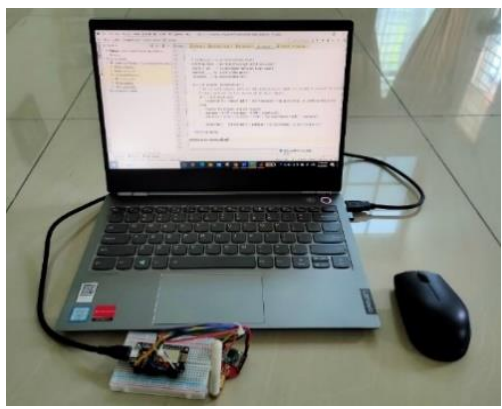

(b)

Figure 3. These figures are; (a) transceiver ESP32-LoRa with BLE beacons and (b) LoRa receiver connected to a laptop 


\subsection{Distance estimation}

After collecting the RSSI, the distance is estimated. Prior to calculating the distance, two parameters will be used in the linear regression method to determine the $m$ (gradient) and $c$ (intercept) values for each BLE beacon. The first parameter is the RSSI collected at each reference point (black dot), the second is the distance between each beacon and each reference point (black dot), which is calculated using the formula (1).

$$
d_{i}=\sqrt{\left(x-X_{i}\right)^{2}+\left(y-Y_{i}\right)^{2}}
$$

The estimate distance will be determined using the (2) [7], but we could convert this formula equation to a slope-intercept formula equation in this section (3).

$$
\begin{aligned}
& \text { Distance }=10^{((\text {Measured Power-RSSI }) /(10 * N))} \\
& \log \text { Distance }=\frac{1}{10 N}((\text { Measured Power })-(R S S I))
\end{aligned}
$$

All the parameter in formula (2) will be separate and it will have 4 parameters and each of them will convert to the variables and parameters of slope formula equation [22]. The parameters of $\frac{1}{10 N}$ and measured power are the constant value, it can be replaced as $m$ (gradient) and $c$ (intercept) and using slope equation formula to find them. RSSI are the data that is collected and log distance is the estimated distance. In (4) as shows in the formula to estimate the distance.

$$
\log \text { Distance }=m(R S S I)+c
$$

\subsection{Particle filter}

There are five critical steps in the particle filtering process which are: a) generate a bunch of particles, b) predict step, c) update step, d) resample, and e) compute estimate. Step b till step e of particle filter will run in loop which converge as near as possible to the true target state by iteratively sampling the particles with the contracted search scope [23], [24].

a) Generate a bunch of particles

Each particle has an associated weight that represents the probability of that particle being the true position of the user. Create a collection of particles using this equation $\left\{x_{k}^{i}\right\}_{i=1}^{N}$ and set the weight for each particle to $\frac{1}{N}$.

b) Predict step

Noise must be added to the particle's movements in order to have reasonable chance of predicting the user's actual position. Without system model uncertainty, the particle filter will fail to accurately model the probability distribution of our belief about the user's position.

c) Update step

The probability that assigned to each position is called as prior (current probability of this position being the true position), but when a new RSSIs' measurement is coming in, the prior need to multiply with the likelihood (probability of the measured distance being same as the distance of particle to the beacon):

$$
\begin{aligned}
& \text { Posterior }=\frac{\text { likelihood } \times \text { prior }}{\text { normalization }} \\
& \mathrm{P}(\mathrm{x} \mid \mathrm{z})=\frac{\mathrm{P}(\mathrm{z} \mid \mathrm{X}) \times \mathrm{P}(\mathrm{x})}{\mathrm{P}(\mathrm{z})}
\end{aligned}
$$

Each particle has position and weight which assesses how well it matches the measurement. Next, normalizing the weights to let it sum up to one and turn them into a probability distribution where the particles that closest to the true position normally will have a higher weight compared to the particles far from the true position.

d) Resample

Particles with extremely low probability will be discarded and replace them with new particles with higher probability. If no new measurements are received during one of the epochs, it is determined when to resample by calculating what many refer to as the effective $\mathrm{N}$, which approximates the number of particles that truly contribute to the probability distribution. 
e) Compute estimate

The mean of the estimate as the sum of the weighted values of the 5000 particles will be calculated. The equation for this is:

$$
\mu=\frac{1}{N} \sum_{1=1}^{N} w^{i} x^{i}
$$

The $x^{i}$ is represent the $i^{\text {th }}$ number of particles.

\subsection{Trilateration}

There are two information that necessary to be used, first is position of BLE beacons (coordinates point) and the estimate distances. The estimate distances here are calculated by using formula (4) with collected RSSI. Next, both data will be used in trilateration to estimate the position for user [25]. Trilateration formula equation is calculated in matrix form and trilateration algorithm is expressed by the following (7) and (8):

$$
\begin{gathered}
A=\left[\begin{array}{cc}
-2\left(x_{1}-x_{n}\right) & -2\left(y_{1}-y_{n}\right) \\
\vdots & \vdots \\
\vdots & \vdots \\
-2\left(x_{n-1}-x_{n}\right) & -2\left(y_{n-1}-y_{n}\right)
\end{array}\right], X=\left[\begin{array}{l}
x \\
y
\end{array}\right], \\
B=\left[\begin{array}{c}
\left(d_{1}^{2}-d_{n}^{2}\right)-\left(x_{1}^{2}-x_{n}^{2}\right)-\left(y_{1}^{2}-y_{n}^{2}\right) \\
\left(d_{2}^{2}-d_{n}^{2}\right)-\left(x_{2}^{2}-x_{n}^{2}\right)-\left(y_{2}^{2}-y_{n}^{2}\right) \\
\vdots \\
\vdots \\
\left(d_{n-1}^{2}-d_{n}^{2}\right)-\left(x_{n-1}^{2}-x_{n}^{2}\right)-\left(y_{n-1}^{2}-y_{n}^{2}\right)
\end{array}\right]
\end{gathered}
$$

The variables $\mathrm{x}$ and $\mathrm{y}$ shown in the algorithm above represents the position of each beacon (coordinate point value) and the $d$ are representing the estimate distance that is calculated using (4). Thus, the estimated position coordinate of users can be solved using (9):

$$
X=A^{-1} B
$$

After estimating positions with and without the particle filter, the difference of distance between these estimated position and actual position will be calculated. The difference of the distance is called as error which will be used to calculate the root mean square error (RMSE) by using the (10):

$$
\operatorname{RMSE}=\sqrt{\sum_{\mathrm{i}=1}^{\mathrm{n}} \frac{\left((\mathrm{x}-\tilde{\mathrm{x}})^{2}-(\mathrm{y}-\tilde{\mathrm{y}})^{2}\right)}{\mathrm{n}}}
$$

\section{RESULTS AND DISCUSSION}

\subsection{Exploring characteristics of BLE beacon}

There are 3 types of BLE beacons initially that has been selected to use in this system. Among them are April beacon sensor N01, bluetooth BLE beacon EEK-N, BLE micro controller NRF52810 where all these are BLE beacons. The sensitivity of BLE beacon indoor positioning has a major effect on positioning accuracy. Therefore, an investigation is needed to know the features and characteristics of these BLE beacons which are more suitable apply in indoor positioning system. The RSSI that is transmitted by each of these BLE beacons will be collected in every different physical distance. The sampling experiment is on every 1 meter in the location environment $[0-15 \mathrm{~m}]$ will be carried out and collect 1 minute for each point in line of sight (LOS) condition.

From the Figure 4 above, it is known that BLE Beacon EEK-N and April beacon sensor N01 have better performance than BLE micro controller NRF52810. This is because the RSSI from BLE micro controller NRF52810 extremely unstable and fluctuation to distance measured, hence effect to lower positioning accuracy. Thus, it is decided to use bluetooth BLE beacon EEK-N in this positioning experiment. 


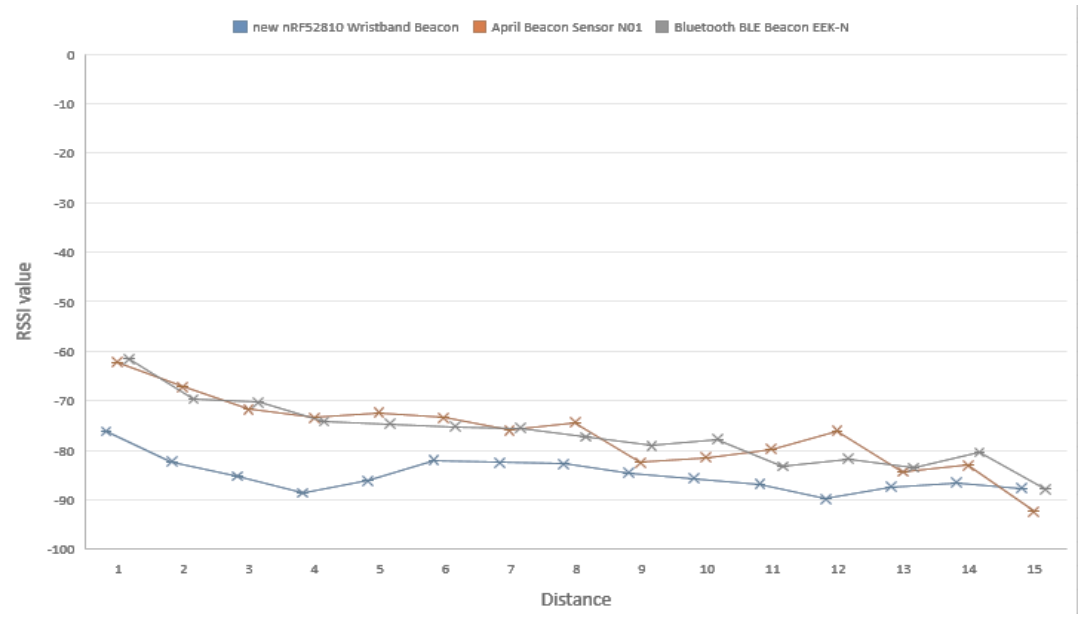

Figure 4. RSSI intensity versus distance for 3 different BLE beacons

\subsection{Testing scenario for the system}

The system is evaluated for accuracy in a variety of different sized environments and different algorithms. Based on previous Figure 4, the maximum signal strength distance that can be considered from beacon to user will be 15 meters. More than that, signal is too weak and cannot be considered in positioning. From this, two scenarios are considered with maximum distance allow and half of the maximum distance. All these two scenarios were conducted in BBNET lab:

- Scenario 1: in this scenario, user will stand at fixed position (coordinate point: $(4,4))$ in 2 difference size area environments in BBNET lab which are $7.5 \mathrm{~m}$ x $8 \mathrm{~m}$ and $15 \mathrm{~m} \times 8 \mathrm{~m}$. Four BLE beacons will be used and deploy at the same position on each corner of the area. The output result from these 2 difference size areas will be compared.

- Scenario 2: $15 \mathrm{~m}$ x $8 \mathrm{~m}$ length area in BBNET lab with 6 beacons used in this scenario. There are 10 fixed output position result of the user around the area will be taken and all error will be presented in CDF for better interpretation.

\subsubsection{Scenario 1}

In experiment 1 referring to Figure 5, it is to observe the effect of difference size of area to the accuracy of the system. Only 4 BLE beacons is used in this experiment. Due to maximum distance vs signal strength measure in Figure 4 is 15 meters, two different size length will be applied here. The first length is maximum capable distance $15 \mathrm{~m}$ by the beacon and half of the maximum which is $7.5 \mathrm{~m}$ length chosen.
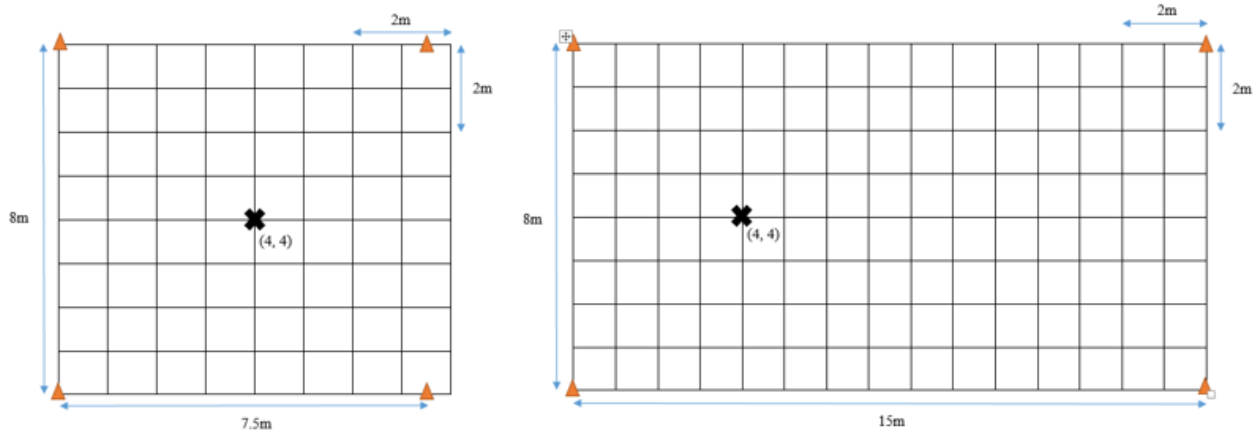

Figure 5. Experiment scenario 1 with $7.8 \mathrm{~m}$ and $15 \mathrm{~m}$ length

Based on bluetooth RSSI data collection, two kind of location estimation algorithm applied. The first one is with particle filter implementation and second without particle filter (basic trilateration). Figure 6 depicts distribution of both algorithm in BBNeT lab for length chosen $7.5 \mathrm{~m}$ while Figure 7 shows the location estimation for length $15 \mathrm{~m}$. 


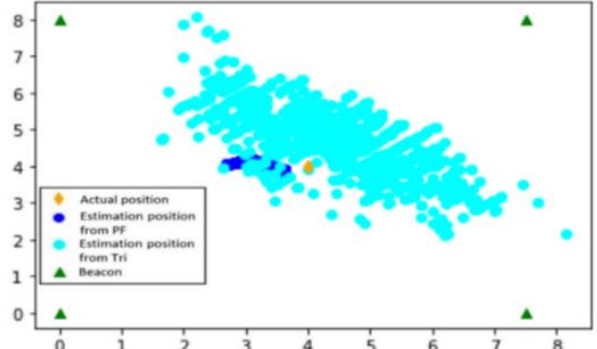

Figure 6. Estimation of user position distribution with and without particle filter in area $7.5 \mathrm{~m} \mathrm{x} 8 \mathrm{~m}$ and $15 \mathrm{~m} \times 8 \mathrm{~m}$

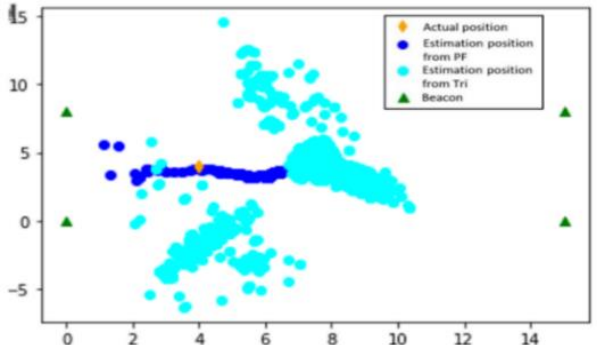

Figure 7. Estimation of user position distribution with and without particle filter in area $15 \mathrm{~m} \times 8 \mathrm{~m}$

In addition, Table 1 as shown in depicts the user actual position, estimated position of the user with and without the particle filter and the root mean square error (RMSE) based on location estimation distribution in Figure 7 and Figure 8. According to the location error RMSE, it is observed that the maximum length distance $15 \mathrm{~m}$ give lower accuracy compared to length area of $7.5 \mathrm{~m}$ for both with and without particle filter implementation. This is because the effective coverage range of BLE beacons is approximately $7.5 \mathrm{~m}$, therefore the RSSI that is transmitted by BLE beacons will attenuate with the increase of the distance between transmitter (BLE beacons) and receiver (ESP32). Besides that, due to BBNET lab environment full of computer, obstacles, and furniture cause the propagation of radio signal may be affected by reflection, scattering, absorption losses and diffraction. Hence, the output result of estimation user position in area size $15 \mathrm{~m} \times 8 \mathrm{~m}$ will be less accuracy compare with area size $7.5 \mathrm{~m} \times 8 \mathrm{~m}$.

Table 1. Estimation of user position and RMSE with and without particle filter

\begin{tabular}{llcc}
\hline & \multicolumn{1}{c}{ Size area } & $7.5 \mathrm{mx} 8 \mathrm{~m}$ & $15 \mathrm{mx} 8 \mathrm{~m}$ \\
\hline \multirow{2}{*}{ Estimated position (average) } & Without particle filter & $(4.46,4.77)$ & $(7.03,3.19)$ \\
RMSE & With particle filter & $(3.61,3.86)$ & $(6.29,3.65)$ \\
& Without particle filter & $1.5683 \mathrm{~m}$ & $4.4375 \mathrm{~m}$ \\
& With particle filter & $0.6907 \mathrm{~m}$ & $2.0557 \mathrm{~m}$ \\
\hline
\end{tabular}

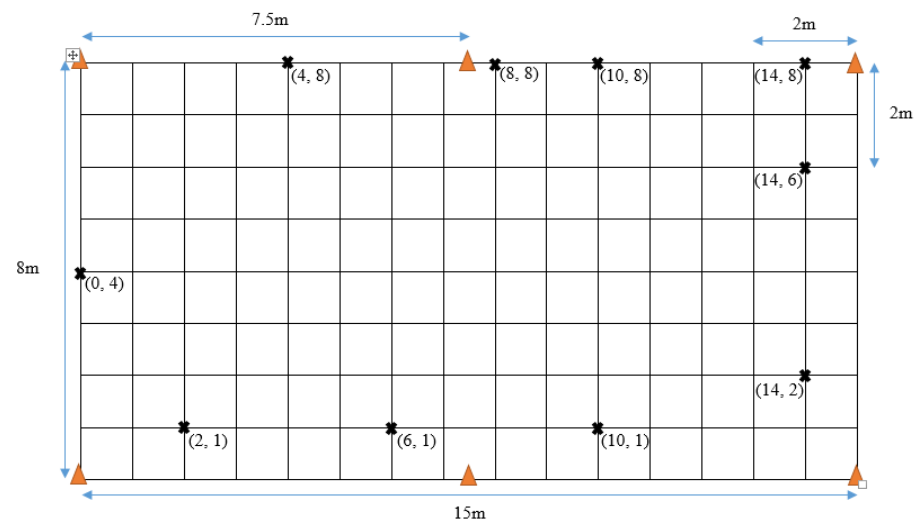

Figure 8. Position measurement on overall location performance in BBNeT lab

\subsubsection{Scenario 2}

The results show in scenario 1 show better positioning result at half of maximum distance between beacon and the user. The area length in the lab is $15 \mathrm{~m}$, so in this case 3 beacons need to be located to maintain distance gap of $7.5 \mathrm{~m}$. In this case, total of 6 beacons need to be deployed to cover the area of $15 \mathrm{~m}$ x $8 \mathrm{~m}$ in BBNeT lab. In this scenario, the user stand at 10 positions in 2 minutes around the $15 \mathrm{~m} \mathrm{x} 8 \mathrm{~m}$ area as depicted in Figure 8. Table 2 depicts the RMSE result from trilateration and particle filter method for each 10 positions. According to result show in the table, most of the point show performance of particle filter is better than trilateration with 6 beacons compared to just 4 beacons. 
Table 2. Estimation of user position and RMSE with and without particle filter

\begin{tabular}{ccccc}
\hline \multirow{2}{*}{ Actual Position of user } & \multicolumn{2}{c}{ RMSE for 4 Beacons } & \multicolumn{2}{c}{ RMSE for 6 Beacons } \\
& Trilateration & Particle Filter & Trilateration & Particle Filter \\
\hline$(2,1)$ & 3.408584399 & 2.057078926 & 7.093784673 & 2.27141599 \\
$(6,1)$ & 2.520563659 & 1.371585559 & 3.599645792 & 2.072848345 \\
$(10,1)$ & 4.401249923 & 4.194079284 & 4.786635796 & 1.958907817 \\
$(14,2)$ & 7.368271665 & 4.626184247 & 2.883902986 & 2.077084072 \\
$(14,6)$ & 3.175313666 & 4.112767608 & 3.009659221 & 2.112709341 \\
$(14,8)$ & 5.943556001 & 5.24396335 & 5.338035218 & 2.11644788 \\
$(10,8)$ & 3.412379156 & 4.291302391 & 1.584292755 & 1.728462153 \\
$(8,8)$ & 4.825274126 & 3.520991851 & 1.89811343 & 2.622271875 \\
$(4,8)$ & 4.831026149 & 1.953638168 & 4.00912834101166 & 3.23589969543883 \\
$(0,4)$ & 5.233537271 & 4.769606473 & 5.860617466 & 2.329960393 \\
\hline
\end{tabular}

Based on all positioning error from scenarios above, comparison have been made with two algorithms, trilateration and particle filter, with 4 beacons and 6 beacons respectively in the cumulative distribution function (CDF). From Figure 9 shows that the estimated position of the user with the particle filter has less error by rely on 6 beacons compare to 4 beacons. Besides, implementing particle filter give significant improvement to trilateration technique. The particle filter method with 6 beacons achieves a $90 \%$ of confident probability of location error within 2.6 meters while basic trilateration gives confident of 6.4 meters ( 6 beacons). Overall particle filter gives $44.44 \%$ improvement over the trilateration method $(6.4 \mathrm{~m})$. By relying on 6 beacons with $7.5 \mathrm{~m}$ aparts, the improvement of CDF is $47.17 \%$ compared to just use 4 beacons with $15 \mathrm{~m}$ aparts.

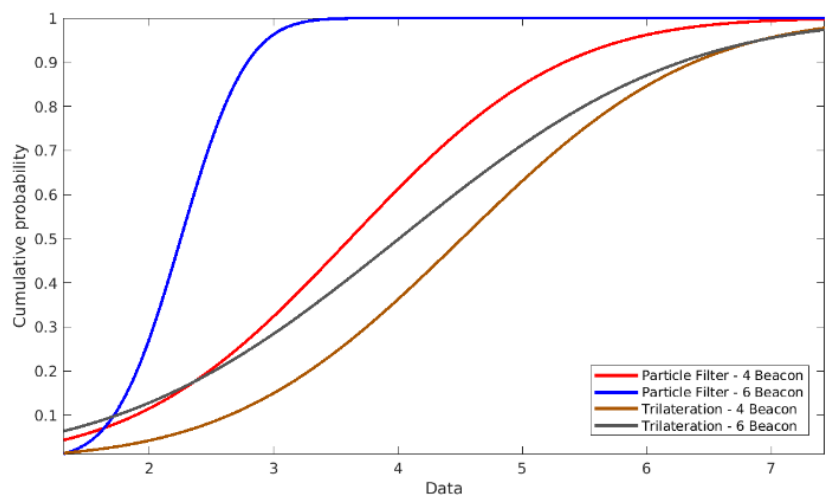

Figure 9. CDF for with and without particle filter

\section{CONCLUSION}

Based on the project carried out, an indoor positioning system has been successfully developed to estimate user position utilizing RSSI that transmitted from the BLE beacons which deployed in BBNeT lab. A transceiver with ESP32 is successfully developed which collect the Bluetooth RSSI and transmit through LoRa to another LoRa receiver. Location estimation algorithm utilizing RSSI data from BLE beacons successfully designed based on trilateration and particle filter implementation. Based on RMSE results, half of the maximum distance give better positioning accuracy compared to maximum distance allow by the beacon itself. Based on this, a simple guideline can be taken in strategy to deployed beacon in BBNeT lab. Finally, the positioning algorithm accuracy in term of RMSE and CDF have been elaborated where the positioning algorithm with implementation of particle filter give accuracy of error less than $2.6 \mathrm{~m}$ for $90 \%$ of the time with improvement of $44.44 \%$ than just basic trilateration. Particle filter with 6 beacons also show significant improvement by $47.17 \%$ compared to just 4 beacons. Future development of this project can be integrated with IoT platform as part of to support the location-based services (LBS).

\section{ACKNOWLEDGEMENTS}

The authors would like to thank the Universiti Teknikal Malaysia Melaka (UTeM), UTeM Zamalah Scheme for sponsoring this research. 


\section{REFERENCES}

[1] A. A. Kalbandhe and S. C. Patil, "Indoor Positioning System using Bluetooth Low Energy," 2016 International Conference on Computing, Analytics and Security Trends (CAST), College of Engineering Pune, India, 2016, pp. 451-455, doi: 10.1109/CAST.2016.7915011.

[2] N. Podevijn, et al., "Performance Comparison of RSS Algorithms for Indoor Localization in Large Open Environments," 2018 International Conference on Indoor Positioning and Indoor Navigation (IPIN), 2018, pp. 1-6, doi: 10.1109/IPIN.2018.8533695.

[3] K. Echizennya and K. Kondo, "Estimation of indoor position and motion direction for smartphones using DNN to BLE beacon signal strength," 2020 IEEE International Conference on Consumer Electronics-Taiwan (ICCETaiwan), 2020, pp. 1-2, doi: 10.1109/ICCE-Taiwan49838.2020.9258282.

[4] D. Giovanelli, E. Farella, D. Fontanelli, and D. Macii, "Bluetooth-Based Indoor Positioning Through ToF and RSSI Data Fusion," 2018 International Conference on Indoor Positioning and Indoor Navigation (IPIN), 2018, pp. 1-8, doi: 10.1109/IPIN.2018.8533853.

[5] P. Barsocchi, A. Crivello, M. Girolami, F. Mavilia, and F. Palumbo, "Occupancy detection by multi-power bluetooth low energy beaconing," 2017 International Conference on Indoor Positioning and Indoor Navigation (IPIN), 2017, pp. 1-6, doi: 10.1109/IPIN.2017.8115946.

[6] G. Pipelidis, N. Tsiamitros, C. Gentner, D. B. Ahmed, and C. Prehofer, "A Novel Lightweight Particle Filter for Indoor Localization," 2019 International Conference on Indoor Positioning and Indoor Navigation (IPIN), 2019, pp. 1-8, doi: 10.1109/IPIN.2019.8911744.

[7] N. A. Azmi, S. Samsul, Y. Yamada, M. F. Mohd Yakub, M. I. Mohd Ismail, and R. A. Dziyauddin, "A Survey of Localization using RSSI and TDoA Techniques in Wireless Sensor Network: System Architecture," 2018 2nd International Conference on Telematics and Future Generation Networks (TAFGEN), 2018, pp. 131-136, doi: 10.1109/TAFGEN.2018.8580464.

[8] D. Plets, N. Podevijn, J. Trogh, L. Martens, and W. Joseph, "Experimental Performance Evaluation of Outdoor TDoA and RSS Positioning in a Public LoRa Network," 2018 International Conference on Indoor Positioning and Indoor Navigation (IPIN), 2018, pp. 1-8, doi: 10.1109/IPIN.2018.8533761.

[9] Z. Jin and X. Zeng, "Research on a Multi-robot Routing Optimization Method Based on Hybrid Lora Location," 2020 5th International Conference on Control, Robotics and Cybernetics (CRC), 2020, pp. 221-225, doi: 10.1109/CRC51253.2020.9253454.

[10] K. Lam, C. Cheung, and W. Lee, "RSSI-Based LoRa Localization Systems for Large-Scale Indoor and Outdoor Environments," in IEEE Transactions on Vehicular Technology, vol. 68, no. 12, pp. 11778-11791, Dec. 2019, doi: 10.1109/TVT.2019.2940272.

[11] W. Choi, Y.-S. Chang, Y. Jung, and J. Song, "Low-Power LoRa Signal-Based Outdoor Positioning Using Fingerprint Algorithm," ISPRS International Journal of Geo-Information, vol. 7, no. 11, p. 440, Nov. 2018, doi: 10.3390/ijgi7110440.

[12] E. Essa, B. A. Abdullah, and A. Wahba, "Improve Performance of Indoor Positioning System using BLE," 2019 14th International Conference on Computer Engineering and Systems (ICCES), 2019, pp. 234-237, doi: 10.1109/ICCES48960.2019.9068142.

[13] H. Li and H. Ma, "A Low Complexity Low Power Indoor Positioning System Based on Wireless Received Signal Strength," 2018 IEEE 20th International Conference on e-Health Networking, Applications and Services (Healthcom), 2018, pp. 1-6, doi: 10.1109/HealthCom.2018.8531137.

[14] M. E. Rusli, M. Ali, N. Jamil, and M. M. Din, "An Improved Indoor Positioning Algorithm Based on RSSITrilateration Technique for Internet of Things (IOT)," 2016 International Conference on Computer and Communication Engineering (ICCCE), 2016, pp. 72-77, doi: 10.1109/ICCCE.2016.28.

[15] S. Naghdi and K. O’Keefe, "Trilateration With BLE RSSI Accounting for Pathloss Due to Human Obstacles," 2019 International Conference on Indoor Positioning and Indoor Navigation (IPIN), 2019, pp. 1-8, doi: 10.1109/IPIN.2019.8911816.

[16] K. Phutcharoen, M. Chamchoy, and P. Supanakoon, "Accuracy Study of Indoor Positioning with Bluetooth Low Energy Beacons," 2020 Joint International Conference on Digital Arts, Media and Technology with ECTI Northern Section Conference on Electrical, Electronics, Computer and Telecommunications Engineering (ECTI DAMT \& NCON), 2020, pp. 24-27, doi: 10.1109/ECTIDAMTNCON48261.2020.9090691.

[17] S. Tasaka, T. Ikari, H. Kaneko, Y. Iijima, R. Yoshino, and M. S. Tanaka, "Study of a bus location system with LoRa in Nonochi city," 2019 IEEE 8th Global Conference on Consumer Electronics (GCCE), 2019, pp. 58-59, doi: 10.1109/GCCE46687.2019.9015321.

[18] S. Okuda and K. Ohno, "Influence of Interference among LoRa Systems under Indoor Environments," 2019 Eleventh International Conference on Ubiquitous and Future Networks (ICUFN), 2019, pp. 16-20, doi: 10.1109/ICUFN.2019.8806114.

[19] J. Klumpe, O. F. Koch, and A. Benlian, "How pull vs. push information delivery and social proof affect information disclosure in location based services," Electron Markets, vol. 30, no. 3, pp. 569-586, 2020, doi:10.1007/s12525-018-0318-1.

[20] H. Torii, S. Ibi, and S. Sampei, "Indoor Positioning and Tracking by Multi-Point Observations of BLE Beacon Signal," 2018 15th Workshop on Positioning, Navigation and Communications (WPNC), 2018, pp. 1-5, doi: 10.1109/WPNC.2018.8555808.

[21] V. C. Paterna, A. C. Augé, J. P. Aspas, and M. P. Bullones, "A Bluetooth Low Energy Indoor Positioning System with Channel Diversity, Weighted Trilateration and Kalman Filtering," Sensors, vol. 17, no. 12, p. 2927, Dec. 2017, doi: 10.3390/s17122927. 
[22] S. Memon, M. M. Memon, F. K. Shaikh, and S. Laghari, "Smart indoor positioning using BLE technology," 2017 4th IEEE International Conference on Engineering Technologies and Applied Sciences (ICETAS), 2017, pp. 1-5, doi: 10.1109/ICETAS.2017.8277872.

[23] H. Luo, et al., "Integration of GNSS and BLE Technology With Inertial Sensors for Real-Time Positioning in Urban Environments," in IEEE Access, vol. 9, pp. 15744-15763, 2021, doi: 10.1109/ACCESS.2021.3052733.

[24] N. Pakanon, M. Chamchoy, and P. Supanakoon, "Study on Accuracy of Trilateration Method for Indoor Positioning with BLE Beacons," 2020 6th International Conference on Engineering, Applied Sciences and Technology (ICEAST), 2020, pp. 1-4, doi: 10.1109/ICEAST50382.2020.9165464.

[25] M. F-I-Azam, Q. Ni and M. Dong, "An Analytical Model of Trilateration Localization Error," 2019 IEEE Global Communications Conference (GLOBECOM), 2019, pp. 1-6, doi: 10.1109/GLOBECOM38437.2019.9013324.

\section{BIOGRAPHIES OF AUTHORS}

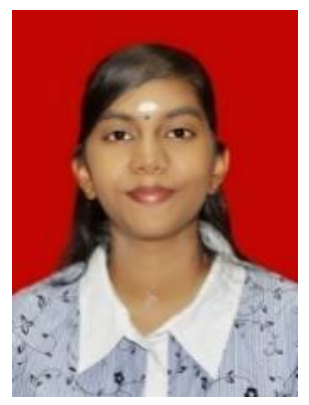

Kavetha Suseenthiran pursued bachelor's degree in Electronic Engineering with honours from UTeM. Currently pursuing Master of Science in Electronic Engineering. Her interest related to indoor positioning with Bluetooth technology and LoRa networks.

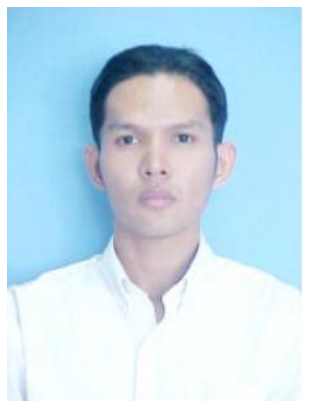

Abd Shukur Ja'afar received both first and master degree from Universiti Teknologi Malaysia (UTM), with Bachelor of Electrical Engineering-Telecommunication (2002). He worked with Samsung electronic in 2003 before completed Master of Engineering in Electronic and Telecommunication (2005). He joined Universiti Teknikal Malaysia Melaka (UTeM) as a lecturer in 2005 and received PhD in Communication System from Lancaster University, UK. Currently his research interest on wireless positioning, indoor positioning and navigation, internet-of-things (IoT), algorithm development with deep learning approach.

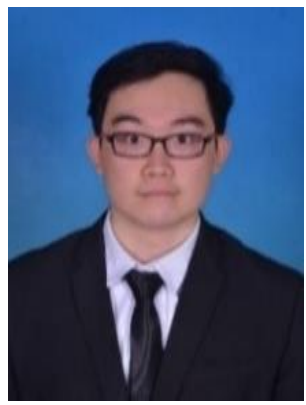

Ku Wei Heng pursued bachelor's degree in Electronic Engineering with honours from Universiti Teknikal Malaysia Melaka (UTeM). Currently pursuing at postgraduate level. His passion is with Bluetooth positioning along with the internet-of-things (IoT) technology.

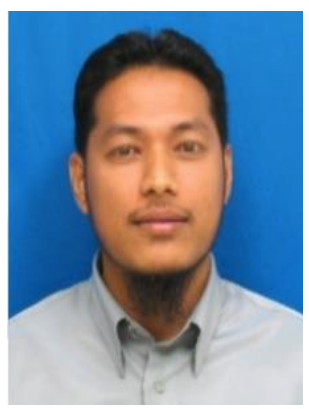

Mohamad Zoinol Abidin Abd Aziz, received both first and master degree from Universiti Teknologi Malaysia (UTM) in Bachelor of Electrical Engineering, and Master of Science in Electronic. He joined Universiti Teknikal Malaysia Melaka (UTeM) as a lecturer in 2005 and received Ph. D in Microwave Engineering in 2015. Currently his research interest on wireless communication system, antenna design and microwave system. 

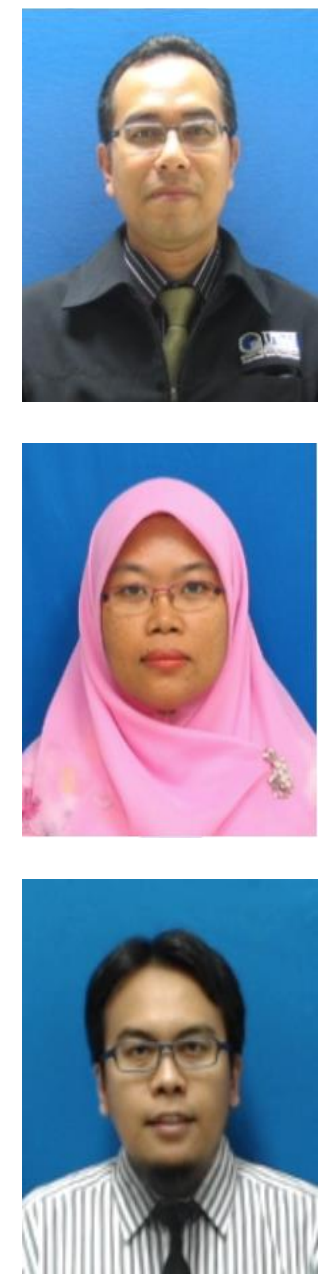

Azmi Awang Md Isa, received the B.S degree from Universiti Teknologi Malaysia (UTM) in 1998, received the M.S degree from Universiti Kebangsaan Malaysia (UKM) in 2004, and obtained his PhD degree in communications systems from Lancaster University, UK in 2011. From 1998 to 2002, he worked as an Engineer with STMicroelectronics. He is currently working as Associate Professor with the Faculty of Electronic and Computer Engineering, Universiti Teknikal Malaysia Melaka (UTeM). His research interests include wireless location technologies, Mobile Radio \& Satellite Communications Navigation Systems.

Siti Huzaimah Husin received the B.Eng (2000) from Multimedia University, M.Eng (2005) from Kolej Universiti Tun Hussein Onn, Malaysia respectively. First appointed as Engineering Instructor (2001) at Kolej Universiti Teknikal Malaysia Melaka and promoted as Lecturer (2005) and Senior Lecturer (2008) in the Department of Industrial Electronics, Faculty of Electronic and Computer Engineering at Universiti Teknikal Malaysia Melaka. Since September 2014, she pursuing PhD in Advanced Control Technology that focused on acoustics energy transfer.

Nik Mohd Zarifie received both first and master's degree from Fukui University Japan, in Electrical-Electronic Engineering-2006 and 2008. He joined UTeM in 2008 and currently pursuing $\mathrm{PhD}$ in computer vision and machine learning. His research interest on image processing with deep learning approach. 